\title{
Au-delà de la confrontation: saisir la diversité des interactions entre mondes militants et mondes économiques
}

\author{
Introduction au dossier \\ "Mondes militants, mondes économiques. \\ Contestations, frontières et coopérations"
}

\begin{abstract}
Laure Bereni (Centre Maurice Halbwachs - CNRS, EHESS, ENS) et Sophie DubuissonQuellier (Centre de sociologie des organisations - CNRS, Sciences Po) ${ }^{1}$

Version autrice.

Pour citer cet article : Laure Bereni et Sophie Dubuisson-Quellier, « Au-delà de la confrontation : saisir la diversité des interactions entre mondes militants et mondes économiques ». Revue française de sociologie, 61(4), 2020, p. 505-29.
\end{abstract}

\section{Résumé}

Depuis une vingtaine d'années, un champ de recherche prolifique a pris pour objet les interactions entre mondes militants et mondes économiques. Cette introduction du dossier spécial "Mondes militants, mondes économiques: contestations, frontières, coopérations" en présente les principaux axes, mais souligne également ses principaux points aveugles. Alors que les travaux dominants du champ "social movement, firms and markets" insistent généralement sur les dimensions de segmentation, de contraste et de confrontation, nous entendons mettre au jour la porosité des frontières entre mondes militants et mondes économiques. Nous proposons trois déplacements analytiques principaux pour saisir les continuités, imbrications et transferts entre ces deux mondes: placer le regard sur des collectifs et pratiques hybrides, situées à l'intersection des mondes militants et économiques; penser en termes d'espaces de cause au sein desquels convergent des acteurs émanant d'univers hétérogènes; déployer une perspective microsociologique, au plus près des militant·es et/ou professionnel·les. En écho avec ces orientations programmatiques, les articles du dossier mettent au jour la grande diversité des interactions entre les mondes militants et économiques, au-delà des logiques de confrontation.

\footnotetext{
${ }^{1}$ Les autrices remercient très chaleureusement Lilian Mathieu et les membres du comité de lecture de la Revue française de sociologie pour leur relecture très attentive de ce texte.
} 


\section{Abstract}

\section{Beyond Confrontation: Capturing the Diversity of Interactions between Activist Worlds and Economic Worlds}

For the past twenty years, a rich body of literature has focused on the interactions between activist and economic worlds. This introduction to the thematic issue "Activist worlds, economic worlds: contentiousness, boundaries, and cooperation" reviews the main insights from this field, but also highlights its blind spots. While the dominant scholarship on "social movements, firms, and markets" insists on the dimensions of segmentation, contrast and confrontation, we call attention to the porosity of the boundaries between activist and economic worlds. We suggest three main analytical shifts to grasp the continuities, overlaps and transfers between these two worlds: 1/a focus on hybrid collectives and practices, at the intersection of the activist and economic worlds; 2/a perspective in terms of "cause fields", within which actors from heterogeneous universes converge ; 3/a micro-sociological approach, centered on the activists and/or professionals. Echoing these programmatic orientations, the articles of this issue bring to light the diversity of interactions between the activist and economic worlds, beyond the logic of confrontation.

\section{Mots clés : mouvements sociaux, acteurs économiques, coopérations, continuités}


Bereni \& Dubuisson-Quellier (2020). Version autrices.

Plusieurs événements survenus en 2020 ont mis au jour les interactions entre contestations sociales et monde des affaires. Au printemps, aux Etats-Unis, la cause de la lutte contre le racisme est projetée au devant de la scène après la circulation sur les réseaux sociaux d'une vidéo montrant l'agonie d'un homme noir, George Floyd, étouffé sous le genou d'un policier blanc. Le drame, qui ravive le mouvement \#BlackLivesMatter né quelques années auparavant, suscite des manifestations massives, en dépit des fortes restrictions liées à la gestion de la crise sanitaire de la Covid 19. Même si le mouvement ne cible pas principalement le monde économique, son onde de choc ne tarde pas à s'y faire sentir. Parfois interpellées directement sur les réseaux sociaux, les plus grandes entreprises étatsuniennes s'engagent publiquement à consacrer des millions de dollars au déploiement de leurs programmes de "diversité et d'inclusion", en dispensant notamment des formations sur le racisme pour leurs salarié $\cdot e^{2}$.

Moins spectaculaires, en France au même moment, de vifs débats s'engagent autour de la responsabilité de la publicité dans le développement d'une société d'hyperconsommation et de ses effets sur le changement climatique. La Convention citoyenne pour le climat, qui réunit 150 citoyennes et citoyens tirés au sort pour faire des propositions en faveur de la transition écologique, demande un encadrement plus fort des pratiques publicitaires, tandis qu'un rapport publié par des organisations militantes dénonce les collusions entre mondes des médias et gros annonceurs. Ce contexte accélère, du côté des professionnels de la communication, les actions visant à inscrire les enjeux de la transition au cœur des pratiques professionnelles, tant pour les annonceurs que pour les publicitaires, à travers la publication de tribunes, la création de groupes de travail, la consultation de parties prenantes ou encore l'organisation d'Etats Généraux de la communication.

Ces événements démontrent la richesse et la diversité des interactions entre mondes économiques et mondes militants, indiquant non seulement que les premiers ne sont nullement ignorants des actions des seconds, mais aussi que les uns et les autres trouvent différentes modalités pour échanger et se confronter sur leurs enjeux et intérêts respectifs. C'est précisément aux interactions entre ces mondes sociaux et à leurs effets que ce numéro thématique est consacré. Cette question fait l'objet d'un champ de recherche foisonnant, souvent dénommé "social movements, firms and markets", qui s'est autonomisé au sein de la sociologie étasunienne depuis les années 2000. Alors que les travaux qui relèvent de ce champ insistent généralement sur les dimensions de segmentation, de contraste et de confrontation, notre dossier propose de mettre au jour la porosité des frontières, les formes de continuité, d'imbrication et de transferts entre mondes militants et mondes économiques. Il s'agit de montrer que les causes ne se construisent pas nécessairement dans des espaces militants autonomes, qui viendraient par des démarches de contestation, les faire accepter par le monde économique. Elles procèdent plutôt de différents types d'échanges aussi bien dans les espaces militants qu'économiques, mais aussi

\footnotetext{
${ }^{2}$ https://builtin.com/diversity-inclusion/companies-that-support-black-lives-matter-social-justice
} 
publics ou médiatiques, selon des logiques allant de la confrontation à la collaboration. Comprendre les contenus de ces causes et les formes prises par les actions visant à les défendre nécessite de rendre justice à ces continuités entre mondes économiques et mondes militants, inscrites dans des espaces nationaux et des contextes temporels spécifiques. Cette porosité entre les mondes militants et les mondes économiques se trouve accentuée depuis quelques décennies, dans un contexte où, d'un côté, les univers militants sont de manière croissante façonnés par des dynamiques d'institutionnalisation, de professionnalisation et de marchandisation, et où, réciproquement, les acteurs économiques se trouvent de plus en plus responsabilisés face aux enjeux de bien commun, sur fond de discours gestionnaires et politiques mettant en équation la poursuite du profit et le déploiement de conduites éthiques par les entreprises.

Nous revenons dans un premier temps sur les apports et les limites de la littérature, principalement nord-américaine, qui a tenté d'éclairer les relations entre mouvements sociaux et économie, pour mettre au jour la manière dont, en mettant beaucoup l'accent sur la confrontation, elle a eu tendance à rigidifier la frontière entre ces deux mondes. Nous proposons ensuite un cadre d'analyse pour identifier certaines formes d'imbrications et de continuités entre mondes militants et mondes économiques, qui peuvent contribuer à expliquer les dynamiques du changement social. Enfin, nous présentons les différents articles de ce numéro en soulignant leurs contributions à la réflexion que nous proposons ici.

\section{Opposition et contestation: les cadres d'analyse dominants des interactions entre mondes militants et mondes économiques}

\section{Les mondes économiques, cibles et arènes de contestation}

Après avoir longtemps centré son attention sur les interactions entre les mouvements sociaux et l'Etat, la sociologie s'est tournée vers l'étude des protestations ciblant les acteurs économiques, à l'échelle des firmes, des marchés ou des secteurs. Cet intérêt nouveau a donné lieu à l'essor du champ de recherche "social movements, firms and markets", fortement marqué par les questions de recherche, les méthodes et les paradigmes qui dominent les trois souschamps de la sociologie étatsunienne dont il s'est principalement nourri: la sociologie des mouvements sociaux, la socio-économie et la sociologie des organisations ${ }^{3}$.

Au départ, ces travaux ont pointé une transformation des mouvements sociaux eux-mêmes, qui se seraient détournés d'actions visant les pouvoirs publics au profit des acteurs marchands,

\footnotetext{
3 Les lignes de force et les frontières du champ de recherche "social movements, firms \& markets", sont régulièrement réaffirmées dans des états de 1'art (Soule, 2012; Walker, 2012) et des dossiers spéciaux de revues (notamment Davis et al., 2008; Fligstein et McAdam, 2019). Pour une mise en perspective des principaux paradigmes de ce sous-champ au sein de la sociologie étatsunienne, voir (Christin et Ollion, 2012).
} 
dans un contexte d'affaiblissement des marges de manœuvre des Etats face aux dynamiques de globalisation des firmes (Vogel, 1996), ou encore en raison de la répression violente de mouvements sociaux dans certains pays (Soule, 2009). Les travaux plus récents sont toutefois revenus sur cette interprétation initiale, en reconnaissant que les mouvements militants ont, de longue date, ciblé tout autant les activités économiques que les Etats (Glickman, 2009). Il importe en outre de ne pas opposer les actions visant les acteurs politiques et celles qui ciblent les acteurs économiques, puisque ces dernières, le plus souvent, ont aussi pour objet de faire évoluer la régulation (Hilton, 2009 ; Chatriot et al., 2006) et sont menées conjointement avec des pressions sur la fabrique de la loi, comme en témoigne la longue trajectoire des luttes syndicales (Fantasia and Stepan-Norris, 2004).

Pour les premiers travaux analysant les interactions entre mouvements sociaux et mondes économiques, il s'agissait de critiquer le caractère stato-centré de la sociologie des contestations collectives (Tarrow, 1994 ; Tilly and Tarrow, 2015) et de montrer que les mondes économiques pouvaient également constituer des cibles ("targets") des mouvements protestataires (Van Dyke, Soule \& Taylor, 2004). Au-delà de ce déplacement analytique, nombre de ces travaux se sont néanmoins contentés de transposer des paradigmes et des cadres théoriques forgés pour analyser les rapports entre mouvements sociaux et Etat, sans profondément les renouveler (Soule 2018; Giugni \& Grasso 2019 ; Walker, Martin et McCarthy, 2008).

Ces recherches ont mis au jour les répertoires d'actions spécifiques qui ciblent les mondes économiques (King et Pearce, 2010). Les analyses se sont d'abord focalisées sur les modes d'action les plus ouvertement contestataires et perturbateurs des routines des entreprises, comme les appels au boycott ou les mises en cause d'entreprises visant à entamer leur réputation (shaming) (King, 2008 ; King and Soule, 2007 ; Bartley and Child, 2010). D'autres travaux ont exploré des démarches plus réformatrices, notamment autour de la mise en œuvre de labels ou de certifications privées (Bartley, 2007 ; Baron, 2013). Certaines recherches ont ainsi montré que des mouvements militants pouvaient aussi contribuer à créer de nouveaux marchés (McInerney, 2014 ; Sine et Lee, 2009 ; Lounsbury et al., 2003), plus ou moins « de niche » (bio, commerce équitable...), voire de nouveaux modèles d'organisations économiques déviant des formes marchandes conventionnelles, comme les coopératives (Schneiberg et al., 2008).

\section{Expliquer et objectiver l'impact économique des contestations}

Au-delà des modalités d'action, une grande partie du champ "social movements, firms and markets" a cherché à identifier les facteurs de la vulnérabilité des acteurs économiques aux contestations sociales qui les visent - et donc les déterminants de l'impact économique (economic outcomes) de ces dernières (Giugni \& Grasso, 2019). Fruit de la transposition du concept de structure d'opportunités politiques issu de la sociologie des mouvements sociaux (McAdam, 1982; Tarrow, 1994), la notion de structures d'opportunités économiques a été mobilisée par plusieurs travaux pour rendre compte de la diversité des réponses des entreprises face à la critique (Schurman, 2004). Joseph Luders (2006), par exemple, a montré le rôle de la 
force des logiques de concurrence, de la position de l'entreprise sur le marché ou encore de l'existence de contre-mouvements pour expliquer l'impact des contestations sociales sur les entreprises. Des recherches centrées sur les dynamiques d'interaction entre les entreprises ont montré que l'adoption d'une pratique par des organisations habituellement résistantes à l'activisme pouvait signaler à d'autres que cette pratique perdait son caractère controversé et qu'elle pouvait ainsi être plus largement adoptée (Briscoe et Safford, 2008). D'autres travaux ont cherché à identifier des facteurs relevant de l'organisation interne des entreprises (Soule \& King 2015). Par exemple, une étude a montré que la propension de firmes pharmaceutiques allemandes à répondre aux pressions émanant du mouvement contre les biotechnologies dépendait des rapports de force au sein du management, en particulier de la concurrence entre cadres exécutifs et cadres scientifiques des départements de recherche et développement Weber et al., 2009).

Plusieurs recherches suggèrent que le succès des contestations sociales visant les acteurs économiques dépend de leur capacité à produire des dommages économiques - ou des anticipations de tels dommages. Tarun Banerjee et Benjamin Case (2020), qui examinent l'impact de campagnes protestataires sur les grandes entreprises étatsuniennes dans la $2^{\text {ème }}$ moitié des années 2000, montrent par exemple que celles-ci ne conduisent à des concessions tangibles (au-delà des «pro-social claims ») de la part des grandes entreprises ciblées que lorsque leurs intérêts financiers sont touchés : les atteintes à leur réputation, notamment dans les médias, s'avèrent bien moins efficaces. De même, pour expliquer pourquoi certaines entreprises sont plus souvent ciblées que d'autres du même secteur, Tim Bartley et Curtis Child (2010) ont pointé que ce sont les actifs économiques, comme une forte valeur boursière et un statut réputationnel élevé, qui constituent paradoxalement leurs points de vulnérabilité.

Focalisés sur la question de l'impact économique des mouvements sociaux, nombre de ces travaux ont privilégié des méthodes qui cherchent à objectiver ces incidences, souvent par des régressions logistiques, au détriment de l'identification de processus plus diffus (Amenta et Polletta, 2019). Par exemple, la question des effets des appels au boycott a donné lieu à force travaux quantitatifs cherchant à mesurer leurs impacts sur le chiffre d'affaires, les actifs financiers, ou le capital réputationnel, en fonction de la médiatisation et des relais dont bénéficient ces appels (King et Soule, 2007 ; King, 2008; DeCelles et al., 2020), mais en négligeant le fait que les militants visaient moins des objectifs purement économiques qu'ils ne cherchaient à ostraciser ou stigmatiser socialement ces acteurs. Il est pourtant indispensable de saisir les effets de ces actions contestataires plus largement sur les dynamiques de concurrence, sur la régulation publique ou privée ou encore sur la notoriété et la légitimité des causes défendues (Dubuisson-Quellier, 2018). Inspirée par les travaux séminaux d'Edwin Amenta et de ses collègues (1992) sur la portée de l'action collective dans les arènes politiques, des recherches ont rendu compte du fait que les mouvements militants pouvaient chercher à activer des mécanismes de médiation pour faire pression sur les entreprises. Ainsi Brayden King (2008) a démontré qu'un appel au boycott était d'autant plus susceptible d'être efficace qu'il était repris largement par les médias. Mais ces médiations ont été essentiellement envisagées comme des 
Bereni \& Dubuisson-Quellier (2020). Version autrices.

modalités stratégiques déployées par les mouvements militants plutôt que comme des mécanismes susceptibles de rendre compte des interactions autour des causes défendues.

\section{Des approches plus dynamiques, constructivistes et contextualisées}

Marquant une certaine distance vis-à-vis de cet agenda de recherche centré sur les déterminants structurels et la mesure de l'impact économique des contestations sociales, d'autres travaux se sont intéressés aux interactions entre mouvements sociaux et entreprises dans une perspective plus dynamique, constructiviste et attentive aux contextes. Tactiques et offensives contestataires des mouvements d'un côté, et réponses et adaptations des entreprises de l'autre, ont été mises en regard l'une de l'autre et analysées comme un jeu séquentiel d'interactions stratégiques. Dans l'étude qu'il a consacrée à la Clean Clothes Campaign, collectif militant luttant contre les pratiques des entreprises du textile en matière de non respect du droit du travail dans les pays d'Asie du Sud-Est, Philip Balsiger (2014) met ainsi en évidence la façon dont les entreprises s'adaptent régulièrement à la critique, ce qui conduit en retour les militantes et militants à adapter leurs stratégies. Etudiant les mises en causes des politiques de greenwashing d'entreprises par l'association écologiste Les Amis de la Terre à travers le prix Pinocchio, Sophie Dubuisson-Quellier décrit la manière dont les interactions entre militants et entreprises conduisent les uns et les autres à négocier les termes et les valeurs de ce qui constitue une pratique économique responsable (Dubuisson-Quellier, 2020). En dépassant l'opposition dichotomique et unidimensionnelle entre réponse et non réponse des entreprises à la critique, les approches interactionnistes ont en outre mis au jour une large gamme de réactions aux dénonciations qui les visent, et souligné les effets que ces réactions peuvent avoir sur les séquences futures de l'interaction. Les entreprises peuvent répondre aux dénonciations par l'ignorance, le déni, ou par différentes techniques de contre-attaque (Balsiger, 2018). Par exemple, elles peuvent tenter de requalifier les critiques à leur avantage, comme ce fut le cas de grandes entreprises européennes donneuses d'ordre mises en cause par des ONG contestataires dans l'affaire de l'effondrement en 2005 d'une fabrique de textile au Bangladesh, au bout de la chaîne de sous-traitance, causant des dizaines de morts et des centaines de blessés graves parmi les ouvrières et les ouvriers: ces entreprises refusèrent de se voir imputer la responsabilité de la catastrophe, et présentèrent le versement d'une aide aux victimes et à leurs familles comme une initiative altruiste, de nature humanitaire (Barraud de Lagerie, 2011). Dans d'autres cas, les entreprises répondent à la critique en discréditant ceux et celles qui la portent par des campagnes de communication ou en les attaquant en justice (Bartley, 2018), ou encore en tentant plus discrètement de les marginaliser, par exemple à travers la création et le financement de nouvelles ONG défendant sur le registre du désintéressement leurs intérêts marchands - une pratique appelée astroturfing (Kraemer et al. 2013; Laurens, 2015).

Dans une perspective nettement critique, des travaux ont étudié les concessions accordées par les entreprises confrontées à la dénonciation sous l'angle de la récupération, de la domestication ou de la managérialisation de la critique. Ainsi, en s'inspirant du cadre néo- 
institutionnaliste, plusieurs recherches ont montré à quel point les "dispositifs de management social" (social management devices) (McDonnell et al., 2015) mis en place pour répondre aux pressions sociales, politiques et juridiques, tels que des dispositifs de RSE, de diversité, ou encore de développement durable, s'apparentent souvent à des "structures purement symboliques" (Edelman, 2016): destinées à signifier la loyauté de l'entreprise vis-à-vis de normes sociales portées par des mouvements sociaux et/ou traduites dans le droit, elles demeurent le plus souvent "découplées" des procédures ordinaires de gestion (Meyer \& Rowan, 1977 ) et sans effets substantiels sur les routines organisationnelles. En outre, souvent appuyées sur des procédures formelles d'auto-régulation (audits, reporting), ces mesures symboliques peuvent faire écran à des contestations futures (Barraud de Lagerie, 2019). Ces réponses des entreprises ont ainsi été étudiées comme des manifestations de la capacité du capitalisme à coopter les critiques qui le visent (Boltanski et Chiapello, 1999) et comme des instruments de légitimation, plus que de déstabilisation, de l'ordre social. Mais l'introduction de ces mesures peut aussi, dans certaines circonstances, favoriser l'exposition des entreprises à la critique, en accroissant leur réputation vertueuse et en élevant dès lors les standards à l'aune desquels elles sont évaluées (Mc Donnell, King et Soule, 2015).

\section{Des outsiders aux insider activists}

Enfin, pour clore le panorama des recherches menées principalement dans le champ étatsunien à l'intersection de la sociologie des organisations et des mouvements sociaux, il importe de mentionner les travaux qui ont placé la focale sur les insider activists, ces personnes qui déploient la critique ou la contestation à l'intérieur des organisations qu'elles ciblent, parallèlement aux outsider activists investis dans les organisations de mouvement social. La notion de militantisme intra-organisationnel a été développée, de manière concomitante, par des travaux sur les mobilisations féministes à l'intérieur des institutions (Eisenstein, 1995; Katzenstein, 1998) et par des recherches critiques en sciences de gestion, depuis les années 1990 (Meyerson \& Scully, 1995). Ces militants et militantes de l'intérieur, qui occupent souvent des positions organisationnelles intermédiaires ou supérieures, portent la critique dans le cadre de leurs activités professionnelles, et se distinguent de ce fait des membres de syndicats qui contestent les conditions d'emploi et de travail dans des sphères dédiées. Par exemple, Debra Meyerson et Maureen Scully (1995) ont proposé d'appeler « radicaux tempérés » (« tempered radicals») les personnes salariées de grandes entreprises privées articulant une loyauté aux normes de leur organisation et à des « idéologies », « causes » ou «communautés » entrant potentiellement en tension avec celles-ci, et qui s'engagent « dans le double projet de travailler au sein de l'organisation et pour changer l'organisation », en dehors des syndicats. Dans une enquête pionnière menée à la fin des années 1990, Nicole C. Raeburn (2004) a montré que les mobilisations pour l'inclusion des couples de même sexe parmi les bénéficiaires des politiques sociales des entreprises ont été conduites non seulement à l'extérieur des organisations, par les associations défendant les droits des gays et lesbiennes, mais aussi, et ce fut un élément crucial de leur succès, "de l'intérieur" par des réseaux de salarié·es gays et lesbiennes au sein des 
grandes entreprises. Si les militantes et militants de l'intérieur sont fortement contraints, dans leurs stratégies d'action, par leur dépendance (financière notamment) à l'organisation qu'ils ciblent, leur position leur donne aussi accès à des ressources dont sont dépourvus les mouvements agissant de l'extérieur (Pettinicchio, 2012), en particulier un niveau élevé d'information sur le fonctionnement et la culture de l'organisation, qui leur permet d'ajuster stratégiquement leurs discours et tactiques (Briscoe et Gupta 2016). Le plus souvent, ces mobilisations se déploient discrètement, "sous le radar", et ne reprennent pas explicitement le langage du militantisme. Pour reprendre les termes de Meyerson et Scully, les tempered radicals se caractérisent par une posture « ambivalente », entre loyauté à la raison managériale et loyauté à un agenda de changement social, par des attentes de changement « modérées » («small wins») et par une aptitude à la flexibilité discursive qui les conduit le plus souvent à «parler la langue des insiders » (Meyerson \& Scully 1995).

\section{Penser les continuités et imbrications entre mondes militants et mondes économiques}

Les travaux du champ "social movements, firms and markets" ont été particulièrement riches et foisonnants au cours des vingt dernières années. Cependant, même lorsqu'ils intègrent des approches plus interactionnistes, constructivistes et contextualisées, ils comportent un certain nombre de points aveugles, en raison de leur arrimage souvent étroit aux paradigmes dominants de la sociologie étatsunienne des mouvements sociaux et des organisations. En particulier, nous soutenons que ces approches tendent généralement à rigidifier la frontière entre les mondes militants et économiques et à forcer les dimensions d'opposition entre ces deux univers, laissant dans l'ombre l'analyse de leurs imbrications et d'autres formes d'interactions. Ce biais est d'autant plus problématique que la période contemporaine est marquée par une porosité croissante de la frontière entre ces deux mondes. Pour restituer toute la richesse et la variété des interactions entre univers militants et économiques, il importe au contraire de décloisonner les cadres d'analyse, en allant puiser davantage dans l'histoire et la sociologie économique, mais aussi dans la sociologie des rapports entre secteur non-lucratif, action publique et sphère marchande.

\section{Décloisonner la sociologie des interactions entre mondes militants et mondes économiques}

Si la littérature anglo-américaine sur les mouvements sociaux et les mondes économiques est très articulée avec la sociologie des organisations, elle se nourrit en revanche assez peu de la sociologie et de l'histoire économiques. Il en résulte souvent une ignorance des mécanismes marchands qui sont en jeu dans ces interactions entre mouvements sociaux et monde économique, comme ceux propres aux marchés, aux dynamiques de concurrence et à la 
régulation économique. Or, plusieurs travaux, souvent menés avec une perspective historienne, ont exploré la relation entre morale et marché, en montrant non seulement que les marchés sont de longue date fortement en prise avec des critiques morales (Fourcade et Healy, 2007; Djelic \& Etchanchu, 2017), mais aussi que ces interactions ne cessent de redéfinir tant les valeurs marchandes que celles de la société (Zelizer, 1979). Ils ont ainsi montré que c'est précisément par ces interactions constantes avec la critique que les marchés se sont donnés des conditions de développement possibles au sein des sociétés, parce qu'y sont négociés de manière récurrente les régimes de valeur et les pratiques marchandes elles-mêmes (Zelizer, 2000). Les marchés contestés ne peuvent se maintenir que grâce à des dispositifs techniques et sociaux, tels que ceux organisant la distribution des jeux d'argent ou du tabac dans des espaces particuliers ou régulés, qui permettent de les rendre acceptables au sein des sociétés où ils se déploient (Steiner et Trespeuch, 2015). L'insuffisante historicisation de la littérature dominante sur les mouvements sociaux et la sphère marchande tend à lui faire oublier le poids des trajectoires longues et symétriquement, à considérer comme spécifiquement contemporaines des formes de mobilisations contre des acteurs économiques ou autour de l'économie, dont les historiennes et les historiens ont au contraire documenté la trajectoire longue. On pense notamment aux travaux sur les mouvements de consommateurs et consommatrices (Chessel, 2012 ; Chessel et Dubuisson-Quellier, 2018) qui ont en outre souligné la forte articulation avec les luttes syndicales (Cohen, 2003 ; Glickman, 2003). Il est symptomatique de constater que les syndicats sont relativement absents de la littérature états-unienne sur les contestations économiques en raison des frontières sous-disciplinaires qui séparent la sociologie des mouvements sociaux de celle portant sur les conflits liés au travail, contribuant à réifier la distinction entre "vieux" et "nouveaux" mouvements sociaux (Sommier, 2011) ${ }^{4}$. Pourtant, les syndicats sont depuis le 19ème des acteurs centraux de la contestation dans les mondes économiques, centrés sur les relations d'emploi et de travail, mais souvent engagés autour de causes sociales et politiques plus larges, qu'ils peuvent aussi contribuer à dynamiser (Isaac et al., 2006).

L'ignorance de l'historicité des rapports entre morale et marchés conduit aussi paradoxalement nombre de travaux à sous-estimer ce qui se joue spécifiquement à l'ère contemporaine. Les travaux sur les transformations du capitalisme, et notamment sur le discours et les dispositifs du néolibéralisme (Amable, 2011; McGoey, 2012), soulignent que ce qui est nouveau depuis quelques dizaines d'années, ce ne sont pas tant les "luttes morales" (Balsiger, 2014) dans les arènes marchandes, ou le recours à des conduites éthiques pour légitimer l'ordre économique, que la prétention désormais ostensible du discours gestionnaire mainstream à fusionner fins morales et fins marchandes, et indissociablement l'affirmation de sa légitimité à s'immiscer dans des domaines qui étaient historiquement monopolisés par des acteurs nonmarchands, en premier lieu l'Etat. Cette nouvelle articulation entre bien commun et marché est

\footnotetext{
${ }^{4} \mathrm{Si}$ les recherches anglophones sur les syndicats et les relations industrielles se sont de plus en plus nourries des paradigmes de la sociologie des mouvements sociaux (Gahan et Pekarek, 2013; Tapia et al., 2015), cette dynamique de décloisonnement n'a pas été réciproque: les syndicats n'ont été que peu intégrés dans le champ des recherches "social movements, firms and markets".
} 
activement soutenue par les pouvoirs publics via, notamment, des labels, des conventions et des dispositions fiscales. Elle se traduit par la diffusion de nouvelles formules dans le monde des affaires (comme celui de "capitalisme conscient", de "shared value" ou de "good capitalism"), par l'institutionnalisation rapide des dispositifs gestionnaires "responsables" et par le développement des fondations d'entreprises. Associations, organisations professionnelles, fondations, syndicats et collectifs d'usagers sont impliqués de manière croissante dans les arènes de discussion d'enjeux de société impliquant les acteurs du monde de l'entreprise, aux côtés des pouvoirs publics. Les syndicats, en particulier, participent aujourd'hui à la définition des politiques RSE des entreprises (Béthoux, 2008; Havard et Sobczak, 2018) et sont engagés sur les questions de transition écologique (Felli et Stevis, 2014). L'essor de ce "nouvel esprit du capitalisme" (Boltanski et Chiapello, 1999) produit des effets sur les rapports entre mondes militants et mondes économiques, qui ont été globalement négligés par la littérature dominante.

À côté des travaux en sociologie et en histoire économique, ce sont les travaux sur les transformations des univers associatifs et de leurs rapports à l'Etat et au marché qu'il convient de mobiliser pour saisir la complexité des interactions entre mondes militants et mondes économiques. Des travaux conduits sur le cas français montrent que depuis une vingtaine d'années les organisations du tiers secteur ont été de manière croissante enrôlées comme opératrices des politiques publiques (Cottin-Marx, Hély, Jeannot \& Simonet, 2017), dans un contexte de réduction de l'emploi public et de redéfinition des modalités de l'action publique, de plus en plus pensées comme un "gouvernement des conduites" (Dubuisson-Quellier, 2016) ou comme un "gouvernement à distance" (Epstein, 2013). Le secteur associatif, traditionnellement décrit comme un lieu d'engagement désintéressé, est ainsi, de plus en plus, un monde du travail (Hély \& Simonet, 2013), auquel est déléguée la prise en charge de problèmes publics: en France, il compte, à la fin des années 2010, 13 millions de bénévoles, mais aussi 1,8 millions de personnes salariées (Tchernonog, 2019). Avec le soutien actif de l'Etat, l'imbrication entre mondes associatif et marchand s'est accrue. Le secteur non-lucratif a été de plus en plus travaillé par des dynamiques de marchandisation, incité à adopter les méthodes gestionnaires issues du privé, et à vendre des produits et des services afin de pourvoir à ses propres ressources. La création par la loi du label de l'Economie sociale et solidaire, en France, a accéléré ce processus d'économisation du tiers secteur en brouillant la frontière entre les organisations historiques de l'économie sociale et des sociétés commerciales affirmant un objectif d'“utilité sociale" (Hély, 2019). Parallèlement, les associations ont été de manière croissante mobilisées par les entreprises dans la conduite de leurs investissements éthiques, qu'il s'agisse de la RSE ou de la philanthropie. Les carrières traversant les frontières entre monde associatif et monde marchand, entre bénévolat et salariat, se sont banalisées (Bory, 2008). Ces transformations des rapports entre univers associatif, monde marchand et action publique ont affecté les rapports entre mondes militants et mondes marchands, dans le sens, comme on va le voir, d'un brouillage des frontières.

Faute d'intégrer les apports de ces différents cadres d'analyse, nombre de travaux inspirés des paradigmes anglo-américains reconduisent une distinction excessivement rigide entre 
mondes militants et mondes économiques, qui elle-même repose sur des oppositions dichotomiques entre outsiders et insiders, entre radicalisme et réformisme, entre perturbation et reproduction de l'ordre social. Cette conception opposant un espace social qui serait celui de la construction de cause et d'expression de la contestation, à un espace économique qui en serait la cible passive, n'est pas tenable. D'une part, dans le contexte contemporain d'institutionnalisation des dispositifs associés au "capitalisme responsable", des acteurs marchands et des groupes professionnels entendent de plus en plus s'investir dans la gestion du bien commun, en discutant de questions d'éducation, de santé publique, d'inclusions sociale et économique, d'environnement, dans les médias, aux côtés des acteurs publics territoriaux, dans des espaces de concertation, en relation avec des organisations politiques. Ces acteurs du monde économique ne représentent pas un ensemble homogène, et s'approprient de manière diverse une grande variété de causes, au-delà des logiques d'instrumentalisation. D'autre part, au sein du secteur nonlucratif, il apparaît de plus en plus difficile de distinguer les acteurs militants, endossant des objectifs clairement politiques et contestataires, d'autres acteurs de la "société civile" qui s'investissent pour des causes sociales en se tenant à distance du langage politique et de la critique frontale de l'économie. L'essor des dispositifs de régulation privée des marchés, et notamment des certifications, a fait converger des acteurs divers, des grandes fondations privées aux organisations non-gouvernementales contestataires (Bartley, 2018 [2007]). Dans des marchés de niche comme celui du bio, du commerce équitable ou des circuits courts, convergent des acteurs très hétérogènes du point de vue du rapport à la critique (LeVelly et al., 2020). Le monde des fondations, qui a connu un fort développement au cours des dernières décennies, est lui-même loin d'être homogène et unifié. Dans certains contextes, des fondations ont pu évoluer au contact d'acteurs plus militants, au point de rallier, de manière inattendue, des mobilisations en faveur d'un Etat social luttant contre les inégalités (Lefèvre \& Berthiaume, 2019). Certes, les collaborations avec les acteurs économiques dominants font débat au sein de l'espace des mouvements sociaux (Mathieu, 2019): les accusations de collusion et de récupération sont fréquentes, et les frontières entre mobilisations radicales et modérées, entre modalités d'action contestataires et institutionnelles, ou encore entre militantisme (activism) et plaidoyer (advocacy) (Siméant, 2014), continuent de faire sens pour les acteurs des mondes militants. Nombre de salarié·es et bénévoles du secteur non-lucratif marquent leur distance à l'égard des dimensions les plus polémiques et critiques du "militantisme politique", au profit d'une posture "d'engagement civique" (Havard-Duclos et Nicourd, 2005; Simonet, 2010). Les conflits de cadrage autour d'enjeux communs (par exemple entre le discours de la "lutte contre les discriminations", associé la critique des inégalités, et celui de la "promotion de la diversité", qui tend à les gommer) traduisent ces clivages au sein de l'univers associatif. Mais ces démarcations entre individus et organisations apparaissent de plus en plus poreuses et labiles. Une même personne peut arborer ou dissimuler le label militant selon les arènes et les contextes d'interactions. Par ailleurs, nombre d'ONG cumulent des modalités d'action institutionnalisées et perturbatrices: elles collaborent avec les entreprises, et parfois leur vendent des services de conseil, tout en participant parallèlement à des actions plus ouvertement critiques, s'appuyant sur 
des modalités plus frontales. Enfin, parmi les organisations engagées dans la défense de causes sociales ou environnementales, le clivage entre modalités contestataires et collaboratives ne reflète pas nécessairement un antagonisme idéologique: une division du travail militant peut s'observer, entre les organisations alignées autour d'objectifs communs, certaines endossant le rôle de good cop et d'autres de bad cop (Bartley, 2018).

\section{Trois déplacements analytiques: collectifs hybrides, espaces de} cause, sociologie des professionnel-les

C'est bien le présupposé d'une frontière discrète et stable entre mondes militants et mondes économiques que nous proposons d'interroger dans ce numéro thématique. A rebours d'une opposition schématique entre contestataires et cibles économiques, nous mettons l'accent sur le continuum des acteurs et des formes de ces interactions. Cela nous conduit à proposer trois déplacements analytiques principaux: 1) placer le regard sur des collectifs et pratiques hybrides, situées à l'intersection des mondes militants et économiques ; 2) penser en termes d'espaces ou de champs au sein desquels des acteurs hétérogènes convergent autour d'un enjeu commun, plutôt qu'en termes de mouvements $v s$. cibles ; 3) déployer une perspective microsociologique, au plus près des professionnel·les - de leurs carrières, de leurs pratiques et de leurs visions du monde.

Premièrement, pour saisir certaines continuités entre mondes militants et mondes économiques, il importe de placer le regard sur des organisations et des pratiques hybrides, qui affirment une dimension de défense de cause tout en épousant les contours de la rhétorique morale du capitalisme, et qui échappent ainsi à une opposition binaire entre contestation et légitimation de l'ordre économique. Cela peut être le cas par exemple de certains groupes professionnels promouvant la finance responsable, la RSE ou la diversité, de cabinets de consultants et bureaux d'experts spécialisés dans la "performance sociale", de fondations, de fonds d'investissement à "impact social", de commissions d'experts sur le développement durable, ou encore d'organisations du secteur de l'Économie sociale et solidaire. Ces acteurs sont engagés dans un travail d'intermédiation entre causes sociales et mondes économiques. On peut les rapprocher des intermédiaires du droit (Talesh \& Pélisse, 2018), d'autant que, le plus souvent, des dispositifs juridiques généralement incitatifs, poussant les entreprises à l'auto-régulation, viennent appuyer les enjeux autour desquels ils orientent leurs activités (dans le cas français, par exemple, obligation de négociation collective sur l'égalité professionnelle, obligation de reporting environnemental pour les entreprises cotées en bourse, "devoir de vigilance", etc.) (Barraud de Lagerie et al., 2020). Ce faisant, ces acteurs hybrides ne font pas qu'importer des causes qui trouveraient leur origine dans les mondes militants. Jouant le rôle de "courtiers" (McAdam et al., 2001) et de "passeurs" (Jobard et al., 2020), ils participent activement à la coconstruction des causes, mais aussi à la fabrication des dispositifs de marché associés à ces causes (tels que des marques ou des catégories marchandes), et plus fondamentalement au façonnement de nouvelles grandeurs marchandes (Dubuisson-Quellier, 2013, 2018 ; Balsiger, 
2016). Ce regard attentif aux acteurs hybrides, à la frontière entre mondes militants et mondes économiques, ne nous conduit en aucun cas à ignorer les clivages, les rapports de force, et les formes de "capture" des causes dans les mondes économiques (Cochoy, 2007). Plusieurs travaux ont documenté la proximité sociale et les convergences d'intérêts entre les membres des fondations, des clubs professionnels, des associations économiques et les strates managériales des grandes entreprises. Des formes de domestication de la critique et de canalisation des protestations ont été mises au jour en particulier par des études sur les pratiques philanthropiques des élites économiques (Duvoux, 2015; Depecker et al. 2018). Jenkins et Halcli (1999), par exemple, ont pointé que les contributions financières des fondations aux mouvements sociaux dans les années 1960-1970 aux Etats-Unis ont orienté les organisations de mouvement social financées vers des modes d'action moins contestataires. Les recherches sur le management de la diversité, par ailleurs, ont mis en lumière le rôle des associations de professionnel·les des ressources humaines dans la construction d'un discours et de pratiques alignant la diversité avec les intérêts managériaux dominants, et mettant à distance les critiques politiques et le cadre juridique de l'antidiscrimination (Dobbin \& Kelly 1998; Bereni 2009). Les travaux d'Élise Penalva Icher (2016) sur les entrepreneurs de l'investissement responsable en France ont souligné la contribution des acteurs dominants de ce secteur émergent à son inscription dans la finance classique, au détriment de visions plus critiques. Mais l'alignement des discours et pratiques de ces acteurs hybrides avec les intérêts économiques dominants, ainsi que leur rôle dans la domestication de la critique et la canalisation de la protestation, ne doivent pas être des présupposés de l'analyse. Ils doivent constituer un objet d'enquête.

Deuxièmement, alors que nombre de travaux reposent sur le présupposé implicite d'une opposition entre, d'une part, le monde des mouvements sociaux et des militants, et de l'autre celui des entreprises et des élites économiques, nous soutenons qu'il peut être heuristique de partir d'une perspective en termes de "champ" ou "d'espace", autour de causes ou d'enjeux (Hoffman, 1999) ayant une forte connotation éthique et de bien commun. Bartley, par exemple, propose d'étudier le champ de la certification dans le domaine de la durabilité qui associe des fondations d'entreprise et des ONG ayant des agendas politiques variés mais convergeant autour d'un instrument orienté vers le marché (Bartley 2018 [2007]). La notion d'espace de cause (cause field) (Bereni 2021), forgée à propos de la cause des femmes, peut également être mobilisée pour l'analyse des configurations de groupes et d'organisations qui orientent durablement leurs activités autour d'une même cause, à l'intersection entre monde économique et d'autres champs sociaux (ONG, acteurs publics, universitaires, cabinets de consultants, organisations professionnelles, fondations, syndicats, think tanks...). Ces champs ou espaces ne sont pas seulement des arènes éphémères, au sens où l'entend Jasper (2019): ils reposent sur des liens durables et sont souvent institutionnalisés, notamment en raison du soutien discret des pouvoirs publics, par le biais de labels et de la commande publique, par exemple. Penser en termes de champs ou d'espaces de causes permet de mettre au jour des dynamiques de convergence et des coalitions possibles entre des acteurs issus d'univers sociaux très variés et relevant d'orientations politiques hétérogènes. Mais cette approche conduit aussi à prêter une 
attention centrale aux clivages, aux rapports de force, et aux conflits entre ces acteurs pourvus de ressources inégales et engagés dans des luttes de cadrage de l'enjeu commun (Bourdieu, 1984; Mathieu, 2019; Fligstein \& McAdam, 2015; Ancelovici 2019).

Troisièmement, alors que la plupart des travaux sur les mouvements sociaux et les mondes économiques sont menés aux échelles macro ou méso-sociologiques, il nous paraît utile de déployer également une perspective microsociologique, au plus près des individus. Il importe en particulier de placer le regard sur des professionnels et professionnelles, qu'il s'agisse des personnes salariées d'ONG, d'organisations du secteur de l'économie sociale, de fondations privées, ou encore du champ de la RSE, en partant de leurs carrières (circulations entre monde économique, secteur non-lucratif et monde administratif), de leurs activités de travail (parfois “sous le radar"), de leurs engagements (des associations professionnelles à des groupes perçus comme militants) ou encore de leurs rapport au travail et au monde (plus ou moins critique). Nous prenons en cela appui sur les pistes fécondes, évoquées plus haut, des travaux sur le militantisme intra-organisationnel, qui montrent que des salarié-es d'entreprises peuvent poursuivre des objectifs politiques dans le cadre de leur travail, et des recherches qui mettent en lumière les circulations entre secteur associatif et monde économique (Simonet, 2010; Lazuech $\&$ Darbus, 2011). Une sociologie au plus près de ces professionnel·les permet de saisir la pluralité des rapports au politique au sein des organisations marchandes comme non marchandes. D'une part, une telle approche permet de se déprendre d'une vision univoque et réifiée des militants, qui sont bien souvent aussi salariés d'associations, engagés dans des carrières professionnelles et des activités économiques, tout autant que dans des mobilisations politiques. D'autre part, des individus travaillant dans le secteur marchand peuvent endosser des perspectives critiques des hiérarchies sociales et se penser comme parties-prenantes d'un mouvement social dans le cadre de leur travail, même si leur activisme est souvent modéré, discret et fortement contraint par l'organisation dans laquelle il se déploie. Les enquêtes récentes sur les responsables des conduites éthiques des grandes entreprises, du développement durable à la diversité, ont ainsi mis au jour la variété de leurs rapports à leur fonction, et leur fréquente ambivalence normative, à rebours d'une opposition nette entre critique et légitimation de l'ordre social (Chiapello \& Gitiaux, 2009; Ghadiri \& al, 2015; Carollo \& Guerci, 2018; Bereni \& Prud'homme, 2019).

\section{Présentation des contributions du numéro thématique}

Ce numéro thématique propose d'explorer une partie des continuités existant entre les mondes économiques et les mondes militants, en identifiant les espaces sociaux et les modalités concrètes de leur mise en œuvre.

Ce sont d'abord des continuités organisationnelles et professionnelles, au sens où chaque catégorie d'organisations, militantes ou économiques, est perméable aux logiques de l'autre. Les espaces dans lesquels se définissent les causes intègrent des enjeux économiques, mais aussi des 
dimensions morales ou des enjeux de bien commun. Différents types d'organisations peuvent ainsi se trouver mobilisées ensemble, dans des démarches qui articulent logiques entrepreneuriales et interventions politiques voire militantes, comme dans le cas de la cause de la lutte contre le gaspillage alimentaire (Mourad, 2018) ou dans celui de le mouvement de l'open data (O’Mahony and Bechky, 2008 ; McInerney, 2014).

C'est l'un des arguments de l'article d'Audrey Chérubin dans ce numéro intitulé "Quand militant-es cyclistes et chauffeurs de bus font cause commune. Une 'improbable convergence' autour de la "mobilité durable à Mexico". En suivant la manière dont des militant·es du déplacement à vélo et des micro-entrepreneurs du transport de bus s'associent autour d'un projet urbain de développement des transports collectifs à Mexico, l'autrice montre que les deux ensembles d'acteurs pourtant bien différents du point de vue de leurs origines sociales et des ressources dont ils disposent vont parvenir à faire cause commune. Cette "improbable convergence" entre l'univers des militant·es cyclistes appartenant à des classes moyennes supérieures urbaines internationalisées et comprenant un nombre élevé de femmes, d'une part, et le milieu professionnel des micro-entrepreneurs du transport de bus formé d'hommes de classe populaire issus de l'exode rural vers la capitale, d'autre part, résulte alors en partie de la manière dont le projet de transport durable permet aux uns et aux autres de développer leur influence, initialement plutôt marginale, dans le secteur des transports. L'autrice met ainsi au jour les dispositions et intérêts des chauffeurs et militant es à la convergence au vu de leurs trajectoires et positions préalables, et accorde une attention particulière aux lieux, dispositifs et agents qui rendent possible la médiation entre les deux mondes, en les replaçant dans une configuration politique, sociale et économique locale. Les logiques entrepreneuriales, qui incitent les microentrepreneurs chauffeurs de bus à accroître leurs positions et à augmenter leurs parts de marché, ne sont pas absentes des stratégies des militants et militantes cyclistes qui se professionnalisent et élargissent leurs ressources. Les deux groupes parviennent alors à s'associer pour favoriser l'extension de politiques publiques de la mobilité durable qui servent leurs intérêts respectifs.

Certaines organisations incarnent directement cette continuité, comme c'est le cas pour les coopératives ou encore les fondations, qui gèrent et produisent des ressources économiques avec des objectifs sociaux.

Dans ce numéro, l'article de Benoît Giry et Pierre Wokuri, intitulé "Une sociologie du rendement social. Le travail d'organisation et ses valeurs dans les coopératives citoyennes d'énergie renouvelable en Bretagne", propose de voir la coopérative comme une organisation articulant performances économiques et militantes. Etudiant différents projets de coopératives de production d'énergies renouvelables en Bretagne, ils s'intéressent au travail de construction d'une organisation productive qui a la particularité de répondre à un besoin social, qu'il s'agisse de l'autonomie énergétique, de la répartition des coûts de l'électricité sur le territoire national, ou encore de la gestion démocratique des politiques énergétiques. Prenant le contrepied des analyses qui considèrent l'articulation entre objectifs productifs et militants comme un jeu à somme nulle, dans lequel tout gain sur un plan induirait mécaniquement des pertes sur l'autre plan, ils 
proposent un modèle dans lequel les logiques productives et militantes s'agencent de manière différenciée selon les activités de l'organisation : les deux peuvent aussi bien se compléter, se substituer l'une à l'autre ou entrer en tension en fonction des contingence de l'activité. Différents modes d'articulations entre objectifs militants et économiques sont susceptibles de coexister au sein de ces organisations, sans nécessairement entrer en tension ou s'hybrider. Du point de vue des acteurs engagés dans ces coopératives, ces différentes valeurs sont toutefois généralement fondues dans un méta-objectif propre, le "rendement social", irréductible à une logique militante ou une logique productive.

Les fondations d'entreprises peuvent aussi se donner à voir comme des organisations intermédiaires entre des univers de causes et le monde des entreprises. Elles sont au cœur de l'article intitulé "Mastercard, sa fondation et l'inclusion financière : une entreprise philanthropique", de Sylvain Lefèvre et Marie Langevin. Mais cette fois, il s'agit plutôt de mettre en évidence les logiques de captation et d'instrumentalisation des causes au profit des intérêts d'une grande firme financière. Ici donc cette continuité entre mondes économiques et mondes des causes est mise au service de la reproduction de l'ordre économique et social, essentiellement. La fondation Mastercard, qui est l'une des plus riches du monde, mène principalement des actions en faveur de l'inclusion financière des populations jeunes en Afrique. Les auteurs montrent que les actions de la fondation, au-delà de la légitimité symbolique qu'elles confèrent à l'entreprise, visent surtout à transformer la société afin d'ouvrir des opportunités marchandes pour la firme, qui dans le cas d'espèce vend des services liés aux technologies de paiement. Pour cela, la fondation assoit sa démarche sur des liens formellement distendus avec l'entreprise, incarnés par la faible présence des membres de l'entreprise au conseil d'administration de la fondation, au profit d'acteurs issus de différents espaces de pouvoir: monde des affaires, mais aussi organisations internationales, gouvernements, universités et grandes ONG. Cette distance formelle de la fondation vis-à-vis de la firme Mastercard lui permet de consolider sa légitimité et son ancrage dans le champ des organisations publiques, privées et non-gouvernementales impliquées dans la lutte contre la pauvreté, et de soutenir ainsi la mise en place d'infrastructures financières et économiques qui vont nourrir la stratégie d'affaire de la firme. Les auteurs proposent le terme "d'entreprise philanthropique" pour qualifier cette architecture particulière de la relation entreprise-fondation, marquée par un jeu subtil de distance et de connexions.

Les continuités se manifestent également entre les modes d'action. En effet, les interactions entre mondes militants et mondes économiques se donnent à voir selon une grande diversité du répertoire de l'action collective des plus conflictuelles aux plus collaboratives (Dubuisson-Quellier, 2018), avec des articulations importantes entre les actions des militants et celles des acteurs économiques.

L'article de Quentin Ravelli, intitulé "Les limbes de la dette : crise économique et mouvements sociaux. Des banquiers de Wall Street aux militants espagnols", apporte sur ce plan une contribution originale. L'auteur analyse la manière dont se déploie en Espagne un 
mouvement social, articulant des organisations engagées dans le droit au logement, dans la défense des consommateurs, dans le mouvement des Indignés ou encore des syndicats et des partis politiques, autour de la question de l'endettement, dans le sillage de la crise des subprimes qui a percuté brutalement l'économie espagnole à la fin des années 2000. Suivant cette question de la dette, depuis les bureaux de Wall Street jusqu'aux cafés dans lesquels se retrouvent les militants et militantes espagnoles pour organiser leur action, Quentin Ravelli met en regard deux types d'activités qui se répondent. D'un côté, les “banquiers" de Wall Street ont déployé depuis les années 1980 un important travail, déjà bien décrit par la sociologie économique autour de la notion de "liquidification" (Carruthers et Stinchcombe, 1999) consistant à titriser la dette pour permettre sa marchandisation dans des espaces financiers internationaux et contribuant à sa circulation et sa désingularisation. Ce mouvement de titrisation de la dette immobilière a été, à l'origine, fortement indexé sur un argumentaire de bien commun (permettre l'accès à la propriété des classes moyennes) et soutenu, à ce titre, par les pouvoirs publics étatsuniens. A l'autre bout de la chaîne de la dette, et plusieurs décennies plus tard, les militantes et militants espagnols opèrent un travail inverse, consistant à partir de différents registres notariaux à ré-assigner les titres de dettes aux individus qui les ont contractés et aux circuits dans lesquels ils circulent. Ils réalisent ainsi un travail de marquage social de l'argent (Zelizer, 1994) contribuant à façonner l'économie de la dette autrement, non plus sur la base de titres devenus liquides et parfaitement anonymisés, mais par une dé-liquidisation de la dette qui permet de réassigner et de tracer des responsabilités parmi les différents opérateurs financiers, depuis les banques espagnoles jusqu'aux acteurs des marchés financiers internationaux. D'une certaine manière ici, c'est bien dans la contestation et l'affrontement que se concrétisent les continuités entre les activités des banquiers et celles des militants, opposant deux visions de l'économie, les deux supposant un important travail social et technique pour attacher des propriétés, soit d'extrême liquidité, soit au contraire de fort marquage social, des titres de la dette.

Dans l'article proposé par Noé Kabouche et Sophie Dubuisson-Quellier intitulé "Militer avec le marché. Les stratégies de collaborations avec les entreprises des associations végétariennes en France", ces continuités entre action militante et action économique se donnent à voir à travers des formes collaboratives. A partir du cas du végétarisme en France, les auteurs montrent comment les organisations militantes contribuent au développement d'une offre marchande de produits végétariens et véganes. Pour cela, elles développent différents outils marchands, comme des labels ou des systèmes de référencement de restaurants, afin d'aider les acteurs économiques à valoriser leurs offres végétariennes. Elles peuvent aussi proposer des prestations de service, souvent non marchandes, pour aider les industriels et les distributeurs à mettre au point ces offres, qu'il s'agisse de trouver des fournisseurs de produits végétariens ou de formuler les produits pour qu'ils plaisent aux consommateurs. L'usage stratégique de ces actions, fortement articulées à d'autres modes d'action, y compris contestataire, permet de comprendre qu'elles font pleinement parties du répertoire de l'action militante. L'article identifie également les conditions sociales de ces collaborations en traçant les porosités très fortes entre ces mondes militants et ces mondes économiques, dessinant un espace social dans lequel salariés et 
bénévoles des organisations militantes et cadres des entreprises sont amenés à se rencontrer et à circuler, favorisant ainsi le déploiement de ces collaborations.

Ces pratiques s'observent aujourd'hui de manière récurrente dans de nombreuses mobilisations. On peut ainsi penser aux prestations proposées par des organisations luttant pour la promotion de la diversité en entreprise pour aider ces dernières à se mettre en conformité avec la loi (Buchter, 2020) ou encore à celles proposées par des ONG autour de services de compensation carbone (Valiergue, 2020). Ces démarches épousent certes les nouvelles contraintes des organisations associatives pour trouver des financements, mais sont aussi stratégiquement articulées à l'action militante puisqu'elles sont envisagées comme des moyens d'enclencher des changements de pratiques de l'intérieur, qu'il s'agisse de développement d'instruments de promotion de la diversité dans le cas étudié par Lisa Buchter ou d'outils de mesure environnementales dans les travaux d'Alice Valiergue, et qu'elles n'excluent pas que les organisations militantes puissent aussi mener des actions de contestation.

Les continuités dans les modes d'actions entre mondes militants et mondes économiques tiennent par ailleurs au fait que les interactions peuvent se jouer sur le temps long, donnant lieu à des séquences d'adaptations mutuelles entre les entreprises et les organisations militantes (Balsiger, 2018). Ces approches longitudinales sont importantes pour comprendre les conditions d'évolution des causes, de leur contenu mais aussi des espaces sociaux dans lesquelles elles se trouvent définies et négociées.

C'est l'une des contributions de l'article de Simon Bittmann intitulé "Comment une entreprise répond à ses critiques ? Une analyse longitudinale du cas Household Finance aux Etats-Unis, 1910-1941", également publié dans ce numéro. L'auteur montre, à partir du suivi sur une longue période, comment une entreprise de crédit s'adapte aux critiques qui la visent. D'une part, on voit que ces interactions doivent moins se saisir comme une opposition entre deux scènes, celles de la critique et celles du monde économique, que comme un jeu à trois, faisant intervenir de manière très significative les pouvoirs publics, dont les dispositions réglementaires arrivent moins pour contraindre les entreprises que pour entériner des évolutions auxquelles ces dernières ont déjà consenti. Cela confirme que le ciblage du monde économique par la critique constitue moins une alternative qu'un moyen d'action envers l'Etat. D'autre part, l'article montre que si la critique a évolué tout au long de cette période, c'est aussi le cas des répertoires d'action développés par l'entreprise pour répondre à la critique. Trois modalités successives sont ainsi identifiées: l'appropriation normative déjà identifiée par la littérature (McDonnell et al., 2015) lorsque l'entreprise intègre les cadres normatifs de la cause; la contribution à la réglementation lorsque l'entreprise s'engage dans une co-production d'un travail réglementaire qui lui est devenu favorable compte tenu de l'évolution de ses pratiques; et enfin la promotion d'une culture consumériste, lorsque l'entreprise s'engage dans une co-production d'une culture de la consommation, très adossée au crédit, qui lui permet de développer des offres intégrant les revendications de la critique. L'histoire qui nous est livrée se donne ainsi moins à voir comme une confrontation récurrente entre militants et entrepreneurs que comme une évolution d'un 
espace de la cause des consommateurs qui se transforme sans cesse à mesure des évolutions de la régulation d'une part et des pratiques et offres des entreprises d'autre part.

La démarche qui est au cœur de ce numéro spécial a clairement des objectifs programmatiques, au-delà d'un bilan de la littérature, déjà bien balisé. Nos propositions théoriques ont pour ambition de mettre au jour une dimension majeure du changement social: les modalités par lesquelles des transformations institutionnelles se produisent. En effet, les espaces sociaux dans lesquels se négocient des enjeux, des problèmes et des causes, tels que la santé publique, le changement climatique, la justice sociale ou l'égalité femmes-hommes, sont le théâtre de confrontations autant que de collaborations qui ont des effets importants sur la manière dont ces questions s'institutionalisent. En suivant les acteurs et les organisations qui y sont impliqués, mais aussi leurs échanges et leurs circulations, leurs modes d'action et leurs instruments, on peut saisir la manière dont ces enjeux sont définis et appropriés, comment ils se confrontent aux normes et aux instruments en place, quels déplacements ils parviennent éventuellement à produire dans les pratiques des acteurs et, en fin compte à quelles transformations sociales et économiques ils donnent lieu.

Nous espérons dans ces propositions dépasser les écueils des visions soit en termes de contestations du monde marchand par la sociologie des mouvements sociaux, soit de moralisation du monde marchand par la sociologie économique, pour suggérer des approches plus dynamiques et interactionnelles du changement social qui prennent en compte les espaces sociaux dans lesquels se définissent et se négocient les enjeux de société.

\section{Bibliographie}

AnCElOVicI M., 2019, " Bourdieu in movement: toward a field theory of contentious politics », Social Movement Studies, p. 1-19. https://doi.org/10.1080/14742837.2019.1637727.

BALsiger P., 2014, The Fight for Ethical Fashion: The Origins and Interactions of the Clean Clothes Campaign, Ashgate Publishing.

BALsiger P., 2018, « Explaining Dynamic Strategies for Defending Company Legitimacy: The Changing Outcomes of Anti-Sweatshop Campaigns in France and Switzerland », Business \& Society, 57, 4, p. 676-705.

BALsiger P., 2016, "Moral Struggles in Markets: The Fight Against Battery Cages and the Rise of Cage-Free Eggs in Switzerland”, European Journal of Sociology, 57,3, p. 419-450.

BARRAUd DE LAGERIE P., 2011, « L'affaire Spectrum. La 'responsabilité sociale des entreprises' à l'épreuve d'un drame », Revue française de sociologie, 52, 2, p. 245-275.

BARRAUd DE LAGERIE P., 2019. Les patrons de la vertu: De la responsabilité sociale des entreprises au devoir de vigilance, Rennes, Presses Universitaires de Rennes. 
Bereni \& Dubuisson-Quellier (2020). Version autrices.

Barraud de Lagerie P., Bethoux E., Mias A., Penalva Icher E., 2020, « La mise en œuvre du devoir de vigilance : une managérialisation de la loi ? \Droit et société, 106, p. 699714.

Bartley T., (2018 [2007]), « Comment les fondations façonnent les mouvements sociaux », Politix, 121, p. 173213.

BARTLEy T., ChILD C., 2014, "Shaming the corporation: Globalization, reputation, and the dynamics of anti-corporate movements", American sociological review, 79, 4, p. 653-679.

BARTLEY T., 2018, Rules without rights: land, labor, and private authority in the global economy, Oxford, Oxford University Press.

BANASZAK L. A., 2010, The women's movement inside and outside the state. Cambridge, New York, Cambridge University Press.

BAnerJeE T., CASE B., 2020, « The Leverage of Protest: Market, Media, and Reputational Disruption in Social Movement Success », Sociological Forum, 35, 1, p. 95-125.

BERENI L., 2009, «'Faire de la diversité une richesse pour l'entreprise': la transformation d'une contrainte juridique en catégorie managériale », Raisons politiques, 35, p. 87-106.

Bereni L., Prud’homme D., 2019, « Servir l'entreprise ou la changer ? Les responsables diversité entre gestion, critique et performance de la vertu ", Revue française de sociologie, 60, 2, p. $175-200$.

BERENI L., 2021, « The women's cause in a field: rethinking the architecture of collective protest in the era of movement institutionalization », Social Movement Studies, 20, 2, p. 208-223.

Bergeron H., Castel P., Dubuisson-Quellier S., 2014, « Gouverner par les labels. Une comparaison des politiques de l'obésité et de la consommation durable », Gouvernement \& action publique, 3, 3, p. 9-32.

Beroud S., 2005, Les Robins des bois de l'énergie, Paris, Le Cherche Midi.

Bethoux E., 2008, « Le comité d'entreprise européen: un acteur de la responsabilité sociale de l'entreprise ? » La Revue de l'Ires, 57, p. 87-110.

Boltanski L., Chiapello E., 1999, Le nouvel esprit du capitalisme, Paris, Gallimard.

BORY A., 2008, « Perdre son âme ou la racheter? Enjeux professionnels et organisationnels autour du bénévolat d'entreprise », Les mondes du travail, 5, p. 39-52. 
Bereni \& Dubuisson-Quellier (2020). Version autrices.

Briscoe F., SAFFord S., 2008, "The Nixon-in-China effect: activism, imitation, and the institutionalisation of contentious practices", Administrative Sciences Quarterly, 53, 3, p. 460491.

Briscoe F., Gupta A., "Social Activism in and Around Organizations ». Academy of Management Annals 10, 1, 2016, p. 671-727.

Bourdieu P., 1984, Questions de sociologie, Paris, Minuit.

BUCHTER L., 2020, " Escaping the Ellipsis of diversity: insider activists' use of implementation resources to influence organisation policy», Administrative Science Quartlery, DOI: $10.1177 / 0001839220963633$.

Carruthers B. G., Stinchcombe A. L., 1999, " The social structure of liquidity: Flexibility, markets, and states », Theory and Society, 28, 3, p. 353-382.

Carollo L., Guerci M., 2018, "'Activists in a Suit': Paradoxes and Metaphors in Sustainability Managers' Identity Work », Journal of Business Ethics, 148, 2, p. 249-68.

Chatriot A., Hilton M., Chessel M.-E., eds, 2006, The Expert Consumer. The History of Retailing and Consumption, Aldershot, Ashgate Publishing, 2006.

Chessel M.-E., 2012, Consommateurs engagés à la Belle Epoque. La ligue sociale d'acheteurs, Paris, Presses de Sciences Po.

Chessel M.-E., Dubuisson-Quellier S., 2018, « The making of the consumer: historical and sociological perspectives », in Kravets Olga, Maclaran Pauline, Miles Steven and Venkatesh Alladi (Eds), The Sage Handbook of Consumer Culture, London, Sage, pp. 43-60.

Chiapello E., GitiauX F., 2009, "Les responsables développement durable des grandes entreprises. Parcours, engagement et représentations", Revue de l'organisation responsable, 4, p. 43-53.

Cochoy F., 2007, « La responsabilité sociale de 1'entreprise comme 'représentation' de l'économie et du droit », Droit et société, 65, p. 91-101.

Cottin-MarX, S., Hely M., Jeannot G., Simonet M., 2017, « La recomposition des relations entre l'État et les associations : désengagements et réengagements ». Revue française d'administration publique, 163, p. 463-476.

Christin A., Ollion E., 2012, La sociologie aux Etats-Unis aujourd'hui, Paris, La Découverte (Repères).

Davis, G. F., Morrill C., Rao H., Soule S. (eds), 2008, " Special issue: Social movements in organizations and markets », Administrative Science Quarterly 53, 3, p. 389-94. 
Bereni \& Dubuisson-Quellier (2020). Version autrices.

Djelic M.-L., Etchangu H., 2017, « Contextualizing Corporate Political Responsibilities: Neoliberal CSR in Historical Perspective », Journal of Business Ethics, 142, 4, p. 641-61.

De BAKKer F., Den Hond F., King B., Weber K., 2013, "Social movements, civil society, and corporations", Organization Studies. 34, 5-6, p. 573-593.

Decelles K.A., Sonenshein S., King B., 2020, “Examining Anger's Immobilizing Effect on Institutional Insiders' Action Intentions in Social Movements", Administrative Science Quarterly, 65, 4, p. 847-886.

Depecker T., Deplaude M. O., Larchet N., 2018, « La philanthropie comme investissement ». Politix, 121, p. 9-27.

Dubuisson-Quellier S., 2013, “A market mediation strategy: How social movements seek to change firms' practices by promoting new principles of product valuation" Organization Studies, 34, 5-6, p. 683-703.

Dubuisson-Quellier S., 2018, « From moral concerns to market values: how political consumerism shapes markets », dans M. BOSTRÖM, M. MICHELETTI, P. OOSTERVEER (di, Boström M., Micheletti M. and Oosterveer P. (Eds.) The Oxford Handbook of Political Consumerism, Oxford, Oxford University Press, pp. 813-832.

DUBUISSON-QUELLIER S., 2020, “Anti-corporate activism and market change: the role of contentious valuations", Social Movement Studies, DOI: 10.1080/14742837.2020.1731448

Duvoux N., 2015, Les oubliés du rêve américain. Philanthropie, État et pauvreté urbaine aux États-Unis, Paris, PUF.

Edelman L., 2016, Working Law: Courts, Corporations, and Symbolic Civil Rights, University of Chicago Press.

EISENSTEIN H., 1995, "The Australian Femocratic Experiment: A Feminist Case for Bureaucracy», dans M. M. Ferree et P. Y. Martin (eds), Feminist Organizations. Harvest of the New Women's Movement, Philadelphia, Temple University Press, p. 69-83.

FANTASIA R., StePAN-NorRIS J., 2004, “The labor movement in motion", dans David A.Snow, Sarah A. Soule and Hanspeter Kriesi (eds), The Blackwell Companion to social movements, Londres, Blackwell, p. 555-575.

Felli R. ET STEvis D., 2014, "La stratégie syndicale d'une transition juste vers une économie durable", Mouvements, 4, 80, p. 111-118.

Fligstein N., McAdam D., 2015, A Theory of Fields. Reprint. Oxford, New York, Auckland, Oxford University Press. 
Bereni \& Dubuisson-Quellier (2020). Version autrices.

Fligstein N., McAdam D., 2019, (eds), "Special issue: Markets and social movements", Socio-Economic Review, 17, 1.

Fourcade M., Healy K., 2007, « Moral views of market society », Annual Review of Sociology, 33, 285-311.

GHADIRI D., GOND J.-P., BrÈs L., 2015, « Identity Work of Corporate Social Responsibility Consultants: Managing Discursively the Tensions between Profit and Social Responsibility », Discourse \& Communication, 9, 6, p. 593624.

Gahan P., Pekarek A., 2013, "Social Movement Theory, Collective Action Frames and Union Theory: A Critique and Extension”, British journal of industrial relations, 51, 4, p. 754776.

GiUgni M., Grasso M. T., 2019, "Economic outcomes of social movements”, dans D. A. Snow, S. A. Soule, H. Kriesi, H. J. McCAmmon (eds), The Wiley Blackwell companion to social movements, Oxford, Wiley Blackwell, p. 466-481.

Glickman, L. B. (2009), Buying power: A history of consumer activism in America, Chicago, IL: University of Chicago Press.

Harvard C. ET SobCzAK A., 2018, “Trade unions and corporate social responsibility : longitudinal analysis of the strategies of French Trade unions Confederations", Industrial Relations, 73, 4, p. 753-783.

Harvard-Duclos B., Nicourd S., 2005, Pourquoi s'engager? Bénévoles et militants dans les associations de solidarité, Paris, Payot.

Hely M., Simonet M. (dir.), 2013, Le travail associatif. Presses universitaires de Paris Ouest.

Hely M., 2019, « Genèse de 'l'entreprise de l'ESS' et désétatisation de l'intérêt général », Informations sociales, 199, p. 88-94.

Hilton M., 2009, Prosperity for all: Consumer activism in an era of globalization, London, Cornell University Press.

Hoffman A. J., 1999, «Institutional Evolution and Change: Environmentalism and the U.S. Chemical Industry ». Academy of Management Journal, 42, 4, p. 351-71.

ISAAC L, MCDONALD S. ET LUKASIK G, 2006, "Taking it from the street: how the sixties mass movement revitalized unionization", American Journal of Sociology, 1, 112, p. 46-96. 
Bereni \& Dubuisson-Quellier (2020). Version autrices.

JASPER J., 2019, « Linking Arenas: structuring concepts in the study of politics and protest », Social Movement Studies, p. 1-15. https://doi.org/10.1080/14742837.2019.1679106.

Jenkins J. C., Halcli A., 1999, «Grassrooting the System? The Development and Impact of Social Movement Philanthropy, 1953-1990 », dans E. C. LAGEMANN (ed.), Philanthropic Foundations: New Scholarship, New Possibilities, Bloomington, Indiana University Press.

Jobard F., Geeraert J., Laumond B., Mützelburg I., Zeigermann U., 2020, « Sociologie politique des passeurs. Acteurs dans la circulation des savoirs, des normes et des politiques publiques », Revue française de science politique, 70, 5, p. 557-573.

King B., PEARCE N., 2010, "The contentiousness of markets: Politics, social movements and institutional change in markets”, Annual Review of Sociology, 36, p. 259-267.

KING B. \& Soule S., 2007, "Social movements as extra-institutional entrepreneurs: The effect of protests on stock price returns", Administrative Science Quarterly, 52, 3, 395-421.

KING B., 2008, "A political mediation model of corporate response to social movement activism", Administrative Science Quarterly, 53, 3, 395-421.

KING B., 2011, "The tactical disruptiveness of movements: Sources of market and mediated disruption in corporate boycotts." Social Problems. 48: 491-517.

Kraemer R., Whiteman G., BanerJee B. « Conflict and Astroturfing in Niyamgiri: The Importance of National Advocacy Networks in Anti-Corporate Social Movements ». Organization Studies 34, 5-6, 2013, p. 82352.

LAZuech G., Darbus F., 2011, « Du militant au manager ? Les “nouveaux” cadres de l'économie sociale » dans P. Bouffartigue (dir.), Cadres, classes moyennes : vers l'éclatement, Paris, Armand Colin, p. 76-86.

LefeVre S., Berthiaume A., 2017, « Les partenariats entre secteur public et fondations philanthropiques au Québec : genèse, contestation et épilogue d'une réforme de l'action publique ». Revue française d'administration publique, 163, p. 491-506.

Le Velly R., Desole M., Chazoule C., " Reprendre la main sur le marché : la construction de circuits intermédiés innovants pour la viande bovine », Revue Française de Socio-Économie, 2020/1 (n²4), p. 129-147.

Lounsbury M., Ventresca M., Hirsch P., 2003, "Social movements, field frames and industry emergence: A cultural-political perspective on US recycling”, Socio-Economic Review, 1,1, p. $71-104$. 
Bereni \& Dubuisson-Quellier (2020). Version autrices.

LOCKE R.M., 2013, The promise and limits of private power: Promoting labor standards in a global economy. New York, NY: Cambridge University Press.

LUDERS J., 2006, “The economics of movement success: Business responses to civil rights mobilization”, American Journal of Sociology, 111, 4, 963-998.

Mathieu L., "The space of social movement", Social Movement Studies, 2019. https://doi.org/10.1080/14742837.2019.1630267

Mourad M., 2018, La lutte contre le gaspillage alimentaire en France et aux Etats-Unis. Mise en cause, mise en politique, mise en marché des excédents alimentaires, Thèse de sociologie de l'Institut d'Etudes Politiques de Paris.

McAdam D., 1982, Political process and the development of black insurgency, 19301970, Chicago, University of Chicago Press.

McAdam D., Tarrow S., Tilly C., 2001, Dynamics of Contention. Cambridge, Cambridge University Press.

MCINERnEY P.-B., 2014, From social movement to moral market, Stanford, Stanford University Press.

McDonnell M.-H., King B., Soule S., 2015, "A dynamic process model of private politics: activist targeting and corporate receptivity to social challenges", American Sociological Review, 80, 3, p. 654-678.

McGoey L., 2012, « Philanthrocapitalism and its critics ». Poetics, 40, 2, p. 185-199.

MeYer J. W., Rowan, B., 1977, « Institutionalized Organizations: Formal Structure as Myth and Ceremony ». American Journal of Sociology, 83, 2, p. 34063.

O’MAHOny S., BECHKY B., 2008, “Boundary Organizations: enabling collaboration among unexpected allies, Administrative Sciences Quarterly, 53, 3, p. 422-459.

PENALVA ICHER É., 2016, « La professionnalisation dans l'Investissement socialement responsable. Le cas des analystes extra-financiers ", Revue française de socio-économie, 16, p. 141-59.

Pettinicchio D., 2012, « Institutional Activism: Reconsidering the Insider/Outsider Dichotomy », Sociology Compass, 6, 6, p. 499510.

SchneIBERG M., King M., \& SMITH T., 2008, "Social movements and organizational form: Cooperative alternatives to corporations in the American insurance, dairy, and grain industries", American Sociological Review, 73, 4, 635-667.

SimÉANT J., 2014, « Interpreting the Rise of International “Advocacy” ». Humanity, 5, 3, p. $323-43$. 
Bereni \& Dubuisson-Quellier (2020). Version autrices.

Simonet M., 2010, Le travail bénévole : Engagement citoyen ou travail gratuit? Paris: La Dispute.

Sine W. \& LEE B., 2009, "Tilting at windmills? The environmental movement and the emergence of the US wind energy sector", Administrative Science Quarterly, 54, 1, 123-155.

Soule S., 2009, Contention and corporate social responsibility. Cambridge, Cambridge University Press.

Steiner P., TRESPEuch M. (dir.), 2015, Marchés contestés. Quand le marché rencontre la morale, Presses universitaires du Midi, Toulouse.

Talesh, S., PÉlisse, J., 2018, How Legal Intermediaries Facilitate or Inhibit Social Change, working papier, LIEPP, Sciences po.

TAPia M., Ibsen C., Kochan T., 2015, "Mapping the frontier of theory in industrial relations: the contested role of worker representation", Socio-economic review, 13, 1, p. 157-184.

TArrow S., 1994, Power in movement, New York, Cambridge University Press.

Tilly C., Tarrow S., 2015, Contentious politics, Oxford, Oxford University Press.

Valiergue A., 2020, Compensation carbone. La fabrique d'un marché contesté, Paris, Sorbonne Université Presses.

VoGel D., 1978, Lobbying the corporation: citizen challenges to business authority, New York, Basic Books.

Vogel D., 2005, The market for virtue: The potential and limits of corporate social responsibility. New York, NY: Brookings Institution Press.

WAlKer, E. T., 2012, "Social Movements, Organizations, and Fields: A Decade of Theoretical Integration », Contemporary Sociology, 41, 5, p. 576-87.

Weber K., Thomas L. G., Rao H., 2009, «From Streets to Suites: How the Anti-Biotech Movement Affected German Pharmaceutical Firms ». American Sociological Review, 74, 1, p. 106-27.

ZELIZER V., 1979, "Human values and the market: The case of life insurance and death in 19th century America”, American Journal of Sociology, 84, 3, p. 591-610.

ZELIZER V., 1994, The social meaning of money. Pin money, paychecks, poor relief and other currencies, New York, Basic Books.

Zelizer V., 2000, Economic livres. How culture shapes the economy, Princeton, Princeton University Press. 